\title{
Biological solutions to aortic root replacement: valve-sparing versus bioprosthetic conduit
}

\author{
Ruggero De Paulis, Raffaele Scaffa, Andrea Salica, Luca Weltert, Ilaria Chirichilli \\ Department of Cardiac Surgery, European Hospital, Rome, Italy \\ Contributions: (I) Conception and design: All authors; (II) Administrative support: All authors; (III) Provision of study materials or patients: All \\ authors; (IV) Collection and assembly of data: All authors; (V) Data analysis and interpretation: All authors; (VI) Manuscript writing: All authors; (VII) \\ Final approval of manuscript: All authors. \\ Correspondence to: Ruggero De Paulis, MD. Department Cardiac Surgery, European Hospital, Via Portuense 700, 00149 Rome, Italy. \\ Email: rdepaulis58@gmail.com.
}

\begin{abstract}
Composite valve graft implantation described by Bentall and De Bono is a well-documented technique of aortic root replacement used for a large spectrum of pathologic conditions involving the aortic valve and the ascending aorta. While mechanical valves were initially used, biological prostheses were later introduced in order to avoid long-term anticoagulation and its related complications. The increasing age of patients who undergo aortic root surgery, and data supporting the use of a biological aortic valve in the younger population, have significantly increased the need for a composite biological valved conduit. However, parallel to the increased use of biological valve in the context of a Bentall operation, aortic valvesparing (AVS) operation have also been performed in a growing number of patients. Sarsam and David described the remodeling and the reimplantation procedures more than 25 years ago with the aim of sparing otherwise normal aortic valves in the presence of a root aneurysm. Important achievements in this discipline have occurred over the past decade including development and refinement of valve preserving aortic root replacement techniques, development of a classification system for aortic insufficiency, surgical approaches to cusp disease with different cusp anatomy. Both procedures can now provide excellent root reconstruction and adequate clinical results in terms of late valve durability. The AVS technique offers several advantages over the Bentall procedure, such as no need for oral anticoagulation and lifestyle adjustments. AVS operations have become established alternatives to Bentall procedures for patients with aortic root pathology. However, data comparing the safety and durability of these approaches are lacking.
\end{abstract}

Keywords: Aortic valve; aortic root; valve sparing; aortic surgery

Received: 12 February 2018; Accepted: 11 April 2018; Published: 09 May 2018.

doi: 10.21037 /jovs.2018.04.12

View this article at: http://dx.doi.org/10.21037/jovs.2018.04.12

\section{Introduction}

The optimal solution for patients presenting with aortic root aneurysms is still a matter of debate.

The "aortic valve-sparing" (AVS) term was coined in the 90s to illustrate procedures developed to spare native aortic valves in patients with aortic root pathology and/or aortic regurgitation. There are conceptually two types of aortic valve sparing operations: remodeling and reimplantation techniques $(1,2)$. The basic concept of both procedures is to eliminate any pathologic dilatation of the root while maintaining normal aortic valve function. In the remodeling technique the Dacron conduit is shaped in three tongues to adapt with the crescent shape of the aortic annulus. Root remodeling recreates the normal configuration of aortic sinuses and sino-tubular junction while the ventricularaortic junction is left untouched. The long-term stability of the procedure has been in fact questioned just because the remodeling lacked annular stabilization. David introduced a different approach called the reimplantation technique, where the aortic valve is spared and sutured within a 
Table 1 Results of Bentall procedures

\begin{tabular}{lccccccc}
\hline First author/year (ref.) & No. & Mean age & $\begin{array}{c}\text { Mean } \\
\text { follow-up }\end{array}$ & $\begin{array}{c}\text { Operative } \\
\text { mortality }\end{array}$ & Survival & \multicolumn{2}{c}{ Freedom from adverse events (at late follow-up) } \\
\cline { 4 - 7 } Hagl 2003 (7) & 142 & 46 ys & 3.5 ys & $0.70 \%$ & $93 \%$ at 8 ys & $100 \%$ & $78 \%$ \\
Etz 2007 (8) & 206 & 53 ys & 5.9 ys & $2.90 \%$ & $89 \%$ at 10 ys & $100 \%$ & $0.7 \times 100$ pts/ys (incidence) \\
Ouzounian 2016 (9) & 180 & 50 ys & 10.7 ys & $0 \%$ & $76.3 \%$ at 15 ys & $80 \%$ & $97.2 \% ; 95.6 \%$ \\
Gaudino 2015 (10) & 421 & 61.5 ys & $\begin{array}{c}16 \text { months } \\
\text { (median) }\end{array}$ & $0.20 \%$ & $81.5 \%$ at 5 ys & $97 \%$ & - \\
\hline
\end{tabular}

ys, years; pts, patients.

straight Dacron graft. Root reimplantation corrects annular ectasia as well as dilatation of the sino-tubular junction. The reimplantation anchors the aortic graft proximally at the ventricular-aortic junction below the leaflets with the commissures sewn inside the polyester fabric graft. However, the major theoretical problem with the classic reimplantation technique is the abolition of both, the sinuses of Valsalva that have a role in regulating leaflet dynamics, and the 3D dynamic motion of the normal aortic annulus. Through the years, thanks to an improved general knowledge, AVS procedures have been significantly refined, so that today is possible to perform a reimplantation technique with the creation of neo-sinuses (3) or a remodeling technique with the addition of annular support $(4,5)$. In this way, both procedures can now guarantee an anatomical root reconstruction and an increased long-term durability.

Nonetheless, the Bentall procedure, whether with a mechanical or a biological valve, still remains the benchmark for treatment of this type of pathology (6). As data accumulate, the advantage of a more conservative approach not requiring an aortic valve replacement became evident especially in the younger group of population. Although the Bentall operation has shown excellent short and long-term results, the question when to replace or spare the valve in the setting of an aortic root aneurysm remains a point of discussion.

Both AVS and root replacement with a biologic composite conduit (bio-Bentall) represent attractive options in aortic root surgery patients willing or needing to avoid anticoagulation. Today, the Bentall interventions with biologic valves are more frequent as are AVS procedures. The question addressed is whether the reimplantation technique or the remodeling technique provide better event free survival than a bio-Bentall procedure. There is not a prospective-randomized study and the data comparing the safety and durability of these approaches are lacking, therefore the answer is not easy.

Patients with an aortic root aneurysm are heterogeneous and the "real" problem is how to compare them in a longterm follow-up. Usually aortic root aneurysms affect the second to fourth decades of life (the dilation starts at the sinuses of Valsalva and progresses towards ascending aorta); instead degenerative ascending aortic aneurysms affect the patients in their fifth to seventh decades of life (the dilation usually starts at the midportion of the ascending aorta and gets on proximally and distally). Second, in the group of patients undergoing a Bentall operation the etiology of aortic valve pathology is more often the stenosis (70\%) than the insufficiency $(30 \%)$. Third, in patients receiving AVS operations the degree of aortic valve regurgitation is rarely severe. Fourth, individuals receiving a bio-Bentall are generally significantly older than those undergoing AVS (thrombo-embolism is often related to comorbidity and the age but not valve type is more often predictive of valverelated mortality). Nonetheless, we attempt to compare, on the basis of the available data, the early and late results of patients undergoing AVS surgery to those undergoing bioBentall operations in terms of operative mortality, survival, valve durability and valve-related complications.

Considering advanced age and higher rate of comorbidities in candidates for a bio-Bentall, we have specifically reviewed studies that analyzed the subset of patients comparable to those who might currently be considered for AVS operations and have a long-term follow-up. The results are shown in Tables 1 and 2.

\section{Operative/in hospital mortality}

\section{Bentall procedures}

In a retrospective analysis, 142 elective patients younger 
Table 2 Results of AVS procedures

\begin{tabular}{|c|c|c|c|c|c|c|c|c|}
\hline First author/year (ref.) & No. & $\begin{array}{l}\text { Mean } \\
\text { age }\end{array}$ & $\begin{array}{c}\text { Mean } \\
\text { follow-up }\end{array}$ & $\begin{array}{l}\text { Operative } \\
\text { mortality }\end{array}$ & Survival & \multicolumn{3}{|c|}{ Freedom from adverse events (at late follow-up) } \\
\hline Yacoub 1998 (11) & 158 & 46 & 5.5 ys & $4.80 \%$ & $89 \%$ at 10 ys & $89.9 \%$ at 10 ys & $64 \%$ (> grade 2 ) & - \\
\hline David 2014 (12) & 371 & 47 & 8.9 ys & $1 \%$ & $76.8 \%$ at 18 ys & $94.8 \%$ at 18 ys & $78 \%$ (> grade 1 ) & $90.1 \%$ at 18 ys \\
\hline Lansac 2017 (4) & 177 & 56 & 41 months & $2.90 \%$ & $89.9 \%$ at 7 ys & $89.5 \%$ at 7 ys & $78 \%$ (> grade 2$)$ & $98.8 \%$ at 7 ys \\
\hline Mastrobuoni 2015 (13) & 187 & 42 & 4 ys & $0.60 \%$ & $94.4 \%$ at 10 ys & $97.4 \%$ at 10 ys & - & $96.6 \%$ at 10 ys \\
\hline Schafers 2017 (5) & 357 & 49 & 57 months & $0.60 \%$ & $81 \%$ at 15 ys & $88.3 \%$ at 15 ys & - & - \\
\hline
\end{tabular}

AVS, aortic valve-sparing; ys, years.

than 65 years without concomitant procedures who underwent Bentall procedure between 1989 and 2000 were studied (7). In this group the $32 \%$ of patients were younger than 40 years of age and the $58 \%$ were between 40 and 60 years (with a mean age of 46 years). The majority of patients were men (85\%). There were no intraoperative deaths. Two patients (1.4\%) had permanent strokes, and 1 of these patients died $(0.7 \%)$.

Often Bentall procedures are performed in the scenario of bicuspid aortic valve (BAV) and significant aortic root dilatation. Etz and colleagues (8) reported the long-term results of Bentall operations performed between 1987 and 2005 in 206 patients with BAV (mean age $53 \pm 14$ years, $84 \%$ male). Aortic regurgitation was present in $53 \%$ of cases while aortic stenosis in $12 \%$ of cases. Twenty-two patients (11\%) underwent urgent or emergent procedures; 11 had acute type A dissection (5\%). Biological valve was implanted in $39 \%$ of patients. Overall hospital mortality was $2.9 \%$, and stroke rate was $1.9 \%$. Risk factors for adverse outcome (death or stroke), which occurred in $4.8 \%$, were presence of clot or atheroma and age older than 65 years.

A recent study compared the outcomes of young patients undergoing aortic root replacement operations (9). In a period of 10 years, a total of 616 patients age $<70$ years and without valvular stenosis underwent elective aortic root surgery (AVS, $n=253$; bio-Bentall, $n=180$; Bentall with a mechanical prosthesis, $n=183$ ). For AVS technique the decision was largely determined by the preference and experience of the surgeon. In bio-Bentall group mean age was $50 \pm 15$ years, $88.9 \%$ were male, and mean follow-up was $10.7 \pm 5.5$ years. BAV was present in 89 patients $(51.7 \%)$ and aortic insufficiency in 171 (95\%). In-hospital mortality and stroke rate were very low ( $0 \%$ and $1.1 \%$ respectively) as we expect in this subset of patients. It must be said that in the same institution, in a most complete analysis performed to date of the bio-Bentall procedures (562 patients), the rate of in-hospital mortality was $2.2 \%$ (14).

Gaudino and colleagues, after examining their results in a cohort of 890 patients operated on from 1997 to 2014, reported that elective aortic root surgery could be performed with very low perioperative risk in high-volume aortic centers. In a cohort of 421 patients that received a bio-Bentall operative mortality was $0.2 \%(10)$. BAV was present in 217 of cases $(51.7 \%$ ) and the aortic insufficiency represented the most common valve status (79.1\%).

\section{Valve-sparing procedures}

The operative mortality of AVS operations is usually low. In the first Yacoub's series of 158 patients undergoing remodeling from 1979 through 1997, the overall mortality was $4.6 \%$ but included 49 emergent procedures for aortic dissection (11).

David reported that in a series of 371 consecutive patients $(78 \%$ men) with a mean age of $47 \pm 15$ years operated from 1988 through 2010 the operative mortality was $1 \%$. In this group $47 \%$ of patients had moderate or severe $\mathrm{AI}(9.2 \%$ with $\mathrm{BAV}), 35.5 \%$ had Marfan syndrome and $12.1 \%$ had type A aortic dissection. In this series remodeling of the aortic valve was used in only 75 patients while reimplantation in 296 patients (12).

When considering the results of AVS with modifications of the original techniques, whether reimplantation or remodeling, the operative mortality also remains in the low range. Thirty-day mortality was $2.9 \%$ in 177 patients with remodeling and external aortic ring annuloplasty collected from the Aortic Valve repair InternATiOnal (AVIATOR) Registry (4). Preoperative aortic insufficiency grade 3 or 
greater was present in 79 patients $(44.6 \%)$. The valve was bicuspid in 59 patients (33.3\%).

In a multicenter trial at four different Italian cardiac surgery centers a total of 278 patients underwent AVS reimplantation using the Valsalva graft the operative mortality was $1.8 \%$. In a total group of 278 patients, 220 were men (79\%), with a mean age of $56 \pm 15$ years, 42 (15\%) had Marfan syndrome, 31 (11\%) had a BAV, 13 (5\%) had acute aortic dissection, and 136 (49\%) had moderate to severe aortic regurgitation (15).

There were 2 operative deaths (1.6\%) in 124 consecutive patients $(87 \%$ men) with aortic root aneurysm treated by reimplantation with Valsalva graft in a single institution at the European Hospital of Rome (from February 2000 to December 2014). In this series of patients, the mean age was $53 \pm 13$ years. Marfan syndrome and BAV were present in $17 \%$ and $12 \%$ of patients respectively (3).

In-hospital mortality was $0.4 \%$ in 187 consecutive adult patients undergoing elective AVS with the reimplantation technique in the group of Mastrobuoni in Brussels (13). The mean age of this cohort was $42 \pm 16$ years, $81.1 \%$ of patients were male and a bicuspid valve was present in $34 \%$ of cases.

Schafers and colleagues reviewed their cumulative 20 years' experience (5). Between 1995 and 2015, 357 patients (324 male; mean age, $49 \pm 13$ years) underwent combined BAV repair and root remodeling. In 225 instances, a suture annuloplasty was added. Only two patients died accounting for a hospital mortality of $0.6 \%$.

\section{Survival}

\section{Bentall procedures}

In the study of Hagl and colleagues on patients younger than 65 years who underwent Bentall procedures the overall survival was $95 \%$ at 5 years and $93 \%$ at 8 years. The rate of death was estimated as 1.0 per 100 persons per year (7).

During an 18-year interval the same group reported an overall survival of $93 \%$ after 5 years and $89 \%$ after 10 years in 206 patients with BAV pathology (8).

The mean follow-up for the patients who underwent elective bio-Bentall procedure at the Peter Munk Cardiac Centre in Toronto was $10.7 \pm 5.5$ years. The cumulative probability of all-cause mortality and mortality from cardiac cause at 15 years were $23.7 \% \pm 4.0 \%$ and $10.2 \% \pm 2.9 \%$, respectively (9).

In the study group of the Weill Cornell Medical College the long-term survival was $81.5 \%$ at 5 years (10).

\section{Valve-sparing procedures}

David reported excellent clinical outcomes in patients with aortic root disease with and without aortic valve regurgitation in a cohort of patients observed prospectively since 1988. At 18 years of follow-up survival was $76.8 \% \pm 4.31 \%$, lower than that for the general population matched for age and gender (12).

Our single center experience showed an overall survival at 5,10 , and 13 years of $94.4 \% \pm 2.2 \%, 90.5 \% \pm 4.4 \%$, and $81.4 \% \pm 7.3 \%$, respectively (3).

Late mortality risk was $5.6 \%$ at 10 years in the cohort of Bruxelles (median duration of follow-up was 4 years) (13).

Lansac and colleagues in 177 patients with remodeling and external aortic ring annuloplasty reported a survival at 7 years of $89.9 \%$ (4).

In the experience of Schneider and colleagues, survival at 15 years was $81 \%(5)$.

\section{Valve durability (freedom from reoperation)}

\section{Bentall procedures}

During the entire follow-up in the series of patients of Mount Sinai Medical Center no patient required reoperation because of a prosthetic dysfunction $(7,8)$.

In the study reported by Ouzounian the probability of reoperation in a young patient receiving a bioprosthesis was $20 \%$ at 15 years of follow-up (9).

Gaudino and colleagues reported that 13 patients (3\%) of the bio-Bentall series underwent reoperation on the aortic valve. However, his median follow-up time was 16 months only (10).

\section{Valve-sparing procedures}

In a quarter of a century of experience with AVS operations David reported at 18 years a freedom from aortic valve reoperation of $94.8 \% \pm 2.0 \%$. Eighteen patients developed aortic regurgitation greater than mild. Freedom from aortic regurgitation greater than mild at 18 years was $78.0 \%$ $\pm 4.8 \%$ (12).

Lansac reported for the whole series freedom from valve-related reoperation of $89.5 \%$ at 7 years. Since 2007 , systematic use of calibrated expansible ring annuloplasty, later followed by systematic cusp effective height 
assessment, significantly increased freedom from valverelated reoperation up to $99.1 \%$ (4).

In our own report, the long-term results of reimplantation with Valsalva graft showed a freedom from reoperation of $95.4 \% \pm 2.3 \%$ at 5 years and $90.1 \% \pm 4.3 \%$ at 13 years. In particular all patients undergoing reoperation for aortic valve regurgitation had surgery during the first 5 years of experience. In fact, freedom from residual aortic regurgitation among the 3 periods was different. Particularly, at 5 years freedom from aortic regurgitation was $89.6 \%, 97 \%$ and $98 \%$ for the first, second, and third period of experience, respectively (3).

Mastrobuoni reported aortic valve reoperation for recurrent severe regurgitation in $2.6 \%$ of patients for a linearized rate of reoperation of $0.6 \%$ for patient-year in 187 consecutive adult patients undergoing elective AVS (13).

In 355 BAV patients Schafers and colleagues reported that reoperation became necessary for recurrent aortic regurgitation in 24 (6 patients underwent reoperation for stenosis). Cumulative incidence of reoperation at 15 years was $21.7 \%(5)$.

\section{Valve-related complications (thrombo-embolism, endocarditis)}

\section{Bentall procedures}

Hagl and colleagues reported an event-free survival (endocarditis, stroke, bleeding and thromboembolism) of $85 \%$ at 5 years and $78 \%$ at 8 years in a selected series of Bentall procedures (elective patients younger than 65 years) (7).

In a median follow-up time of 5.9 years $(1,200$ patientyears) for patients with BAV undergoing Bentall procedure at Mount Sinai Medical Center the linearized stroke rate was 0.7 per 100 patient-years (8).

In the 180 selected patients undergoing a bio-Bentall procedure reported by Ouzanian and colleagues, longterm rates of endocarditis and thromboembolism (cerebral stroke and transient ischemic attacks) were $2.8 \%$ and $4.4 \%$ respectively.

\section{Valve-sparing procedures}

David reported a freedom from thromboembolic events at various intervals $(92.2 \pm 2.4$ at 15 years and $90.1 \pm 3.2$ at 18 years) (12).

At 7 years of follow-up Lansac and colleagues reported a freedom from thromboembolic events of $98.8 \%$ (4) while Mastrobuoni reported a risk of systemic embolism of $3.4 \%$ at 10 years (13).

\section{Direct comparison [propensity score matching (PSM)]}

In a recent study, 749 consecutive patients underwent either a valve-sparing operation or a root replacement with a biologic composite conduit at two institutions (Weill Cornell Medicine in New York and European Hospital in Rome) (16). PSM was used to compare similar cohorts of patients in the overall population and in the $\leq 55$ and $\geq 65$-year age groups. Overall operative mortality was $0.4 \%$, mean age $57.4 \pm 14.3$ years, $84.6 \%$ were male. Individuals in the biologic composite conduit group were older and had worse preoperative risk profiles. In the unmatched population, there was no difference in in-hospital deaths (0 in the valve-sparing versus 3 in the biologic composite conduit group; $\mathrm{P}=0.12$ ). At a mean follow-up of $27.5 \pm 28.4$ months no significant differences in terms of mortality were found between the two groups ( 2 vs. 0 follow-up deaths in the AVS and in the bio-Bentall groups, respectively; $\mathrm{P}=0.11$ ). During the follow-up, more patients in the biologic composite conduit group underwent reoperation on the aortic valve $(2.6 \%$ vs. $1.5 \% ; \mathrm{P}=0.026)$ resulting in a freedom from reoperation of $97.4 \%$ vs. $98.5 \%$, respectively. The PSM process resulted in 145 pairs of patients with almost identical baseline characteristics. No differences between groups were found. No significant differences in postoperative survival were found between the matched series. PSM identified 71 and 44 pairs of patients respectively in the younger and older age group who received an AVS or a bio-Bentall. In-hospital outcomes were substantially similar for both procedures in both age groups before and after PSM. No difference in survival at follow-up was found between groups stratified by age after matching. No difference in reoperation rate at follow-up was found between groups stratified by age. This report concluded that in case of aortic root aneurysm, both valvesparing operations and root replacement with a biologic composite conduit provide excellent outcomes. However, at mid-term follow-up the use of biologic composite conduit was associated with a higher risk of reoperation.

As mentioned previously, Ouzounian reported the results of a direct comparison between patients undergoing AVS or Bentall operations (9). After the use of a propensity score and adjusting for clinical covariates, bio-Bentall procedures 
were associated with increased long-term major adverse valve-related events compared with patients undergoing AVS [hazard ratio (HR): 3.4, $\mathrm{P}=0.005$ ] and increased cardiac mortality (HR: 7.0, $\mathrm{P}=0.001$ ). Furthermore, bioBentall procedures were associated with increased risk of reoperations (HR: 6.9; $\mathrm{P}=0.003$ ) compared with AVS procedures.

\section{Conclusions}

Nowadays, the options for root surgery are mechanical valved conduits, biological xenograft valved conduits (with stented or stentless valve), homograft, autograft or valvesparing procedures. However, each patient should be individually evaluated. Several factors have to be taken into consideration when choosing the type of operation or the prosthetic heart valve (age of the patient, cardiac and noncardiac comorbidity). Thromboembolism, valve durability and endocarditis are the major causes of valve-related late postoperative morbidity and mortality. Thromboembolism and bleeding rate are higher in patients with mechanical valves comparing to the ones with biological valves while the incidence of endocarditis is similar in both groups $(17,18)$. On the other hand, preserving the natural aortic valve is expected to greatly decrease most of these complications.

In the last years a better understanding of benefits and risks of the various types of prostheses has resulted in a growing number of patients, even in a younger population, who prefer to choose a "biologic solution". The reasons for this trend vary. First, patients undergoing aortic valve surgery are interested not only in life expectancy but also in quality of life. To this extent, anticoagulant therapy is considered a huge limitation to quality of life, especially in those young patients. Another important aspect is the perception that the most recent prostheses available will last longer because they are designed with new technologies and anti-calcification treatments. Finally, the valve-invalve approach using endovascular techniques may offer an effective, less invasive treatment for patients with valvular dysfunction of bioprostheses and, therefore, the possibility of overcome the problem of structural valve deterioration (SVD) without the need for a surgical reoperation.

Sarsam (1) and David (2) introduced AVS procedure, which has the great advantage of eliminating the need for lifelong anticoagulation therapy and preventing prosthetic valves-related risks. AVS procedures have gained popularity in recent years because of a better understanding of aortic root anatomy and physiology. In the quest for an optimal root reconstruction, through the years, several modifications have been proposed to the original reimplantation or the remodeling technique. All largest series on results of AVS procedure invariably report an operative mortality extremely low, ranging from $0 \%$ to $5 \%$, with a ten-year survival around $90 \%$, definitely establish it as a safe, effective and reproducible technique. The major aspect to be clarified remains the long-term durability of the spared valve in terms of incidence of residual aortic valve regurgitation and the consequent need for AVR in the subsequent years. The majority of available data on the 10 years follow-up show a freedom from reoperation varying from $87 \%$ to $95 \%$ with a freedom from significant residual aortic insufficiency around $90 \%$. Small differences in the results might depend from the single center experience or the used technique. However, the results are generally satisfactory especially considering the relatively "young age" of this procedure.

Historically, the main advantage of the reimplantation was a resulting annular stabilization with the disadvantages of an unfavorable hemodynamic. Conversely, the remodeling offers a better physiological hemodynamic and a simple and shorter surgical technique, while the disadvantage remained in the lack of annular support. With technical advances and modifications, however, the differences between these two procedures have narrowed and both can offer equal safety and reproducibility.

Despite in the most comprehensive analysis performed to date of the root surgery, early post-operative outcomes and overall long-term survival were similar between AVS and bio-Bentall interventions, AVS procedures seem to be associated with improved long-term freedom from cardiac death. Furthermore, the "spared valve" (being native living tissue) seems to be more resistant to endocarditis and with improved freedom from reoperations as compared to patients undergoing bio-Bentall procedures. If the aortic valve can be spared, AVS procedures should be considered for patients undergoing aortic root replacement. If sparing is not possible or challenging, the Bentall procedure offer similar low operative risk and good long-term results. A minimum follow-up of 15 years is necessary to evaluate both the incidence of SVD for biological aortic valve or the good functioning of a spared valve.

\section{Acknowledgements}

None. 


\section{Footnote}

Conflicts of Interest: The authors have no conflicts of interest to declare.

\section{References}

1. Sarsam MA, Yacoub M. Remodeling of the aortic valve anulus. J Thorac Cardiovasc Surg 1993;105:435-8.

2. David TE, Feindel CM. An aortic valve-sparing operation for patients with aortic incompetence and aneurysm of the ascending aorta. J Thorac Cardiovasc Surg 1992;103:617-21.

3. De Paulis R, Chirichilli I, Scaffa R, et al. Long-term results of the valve reimplantation technique using a graft with sinuses. J Thorac Cardiovasc Surg 2016;151:112-9.

4. Lansac E, Di Centa I, Sleilaty G, et al. Remodeling root repair with an external aortic ring annuloplasty. J Thorac Cardiovasc Surg 2017;153:1033-42.

5. Schneider U, Feldner SK, Hofmann C, et al. Two decades of experience with root remodeling and valve repair for bicuspid aortic valves. J Thorac Cardiovasc Surg 2017;153:S65-71.

6. Bentall H, De Bono A. A technique for complete replacement of the ascending aorta. Thorax 1968;23:338-9.

7. Hagl C, Strauch JT, Spielvogel D, et al. Is the Bentall procedure for ascending aorta or aortic valve replacement the best approach for long-term event-free survival? Ann Thorac Surg 2003;76:698-703; discussion 703.

8. Etz CD, Homann TM, Silovitz D, et al. Long-term survival after the Bentall procedure in 206 patients with bicuspid aortic valve. Ann Thorac Surg 2007; 84:1186-93; discussion 1193-4.

9. Ouzounian M, Rao V, Manlhiot C, et al. Valve-sparing root replacement compared with Composite valve graft

doi: 10.21037/jovs.2018.04.12

Cite this article as: De Paulis R, Scaffa R, Salica A, Weltert L, Chirichilli I. Biological solutions to aortic root replacement: valve-sparing versus bioprosthetic conduit. J Vis Surg 2018;4:94. procedures in patients with aortic root dilation. J Am Coll Cardiol 2016;68:1838-47.

10. Gaudino M, Lau C, Munjal M, et al. Contemporary outcomes of surgery for aortic root aneurysms: A propensity-matched comparison of valve-sparing and composite valve graft replacement. J Thorac Cardiovasc Surg 2015;150:1120-9.e1.

11. Yacoub MH, Gehle P, Chandrasekaran V, et al. Late results of a valve-preserving operation in patients with aneurysms of the ascending aorta and root. J Thorac Cardiovasc Surg 1998;115:1080-90.

12. David TE, Feindel CM, David CM, et al. J Thorac Cardiovasc Surg 2014;148:872-9; discussion 879-80.

13. Mastrobuoni S, Tamer S, de Kerchove L, et al. Valve sparing: aortic root replacement with the reimplantation technique. Multimed Man Cardiothorac Surg 2015;2015. doi: $10.1093 / \mathrm{mmcts} / \mathrm{mmv} 012$

14. Ouzounian M, Rao V, Manlhiot C, et al. David vs. Goliath: valve-sparing root replacement improves outcomes compared to Bentall procedures in patients with aortic root dilatation. Can J Cardiol 2015;31:S218-9.

15. De Paulis R, Scaffa R, Nardella S, et al. Use of the Valsalva graft and long-term follow-up. J Thorac Cardiovasc Surg 2010;140:S23-7; discussion S45-51.

16. Gaudino M, Di Franco A, Ohmes LB, et al. Biological solutions to aortic root replacement: valve-sparing versus bioprosthetic conduitł. Interact Cardiovasc Thorac Surg 2017;24:855-61.

17. Puvimanasinghe JP, Steyerberg EW, Takkenberg JJ, et al. Prognosis after aortic valve replacement with a bioprosthesis: predictions based on meta-analysis and microsimulation. Circulation 2001;103:1535-41.

18. Khan SS, Trento A, DeRobertis M, et al. Twenty-year comparison of tissue and mechanical valve replacement. J Thorac Cardiovasc Surg 2001;122:257-69. 